\title{
2-S16-1
}

\section{Role of stiffness sensors in skeletal muscle regeneration}

\author{
Yuji Hara ${ }^{1}$, Kotaro Hirano ${ }^{1}$, Yasuo Mori ${ }^{1}$, Masato Umeda ${ }^{1,2}$
}

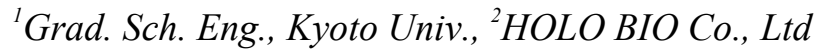

Skeletal muscle has the capacity to regenerate myofibers in response to muscle injury. Upon a variety of stimuli including mechanical stretch, muscle-resident stem cells called muscle satellite cells (MuSCs) are committed to become myoblasts that can fuse with each other to regenerate myofibers. The rigidity of the microenvironment is thought to regulate regeneration of myofibers, but the molecular entity of the stiffness sensor that determines the function of MuSCs remains to be elucidated. In this session, we will present our recent data showing that PIEZO1, a $\mathrm{Ca}^{2+}$-permeable mechanosensitive ion channel that is activated by membrane tension, plays a crucial role in the function of MuSCs. Conditional deletion of the Piezo1 gene in MuSCs leads to delayed myofiber regeneration after muscle injury, at least in part due to a growth defect of MuSCs caused by a reduced formation of actomyosin. Thus, we propose the novel mechanism: $\mathrm{Ca}^{2+}$ influx in response to mechanical stimulation may be involved in adjustment of cellular stiffness that is essential for the MuSC function. 